\title{
BMJ Open Prospective, randomised, controlled study on the efficacy and safety of different strategies of tranexamic acid with total blood loss, blood transfusion rate and thrombogenic biomarkers in total knee arthroplasty: study protocol
}

Yong Yang, ${ }^{1,2}$ Zheng Wang, ${ }^{1,2}$ Faxuan Wang, ${ }^{3}$ Xin Zhao, ${ }^{1}$ Kaijie Yang, ${ }^{1}$ Jinlong He, ${ }^{1}$ Yun Jin, ${ }^{1}$ Haibo Yang, ${ }^{1}$ Dong Ding, ${ }^{2}$ Qunhua Jin (D) ${ }^{1}$

To cite: Yang Y, Wang Z, Wang $F$, et al. Prospective, randomised, controlled study on the efficacy and safety of different strategies of tranexamic acid with total blood loss, blood transfusion rate and thrombogenic biomarkers in total knee arthroplasty: study protocol. BMJ Open 2021;11:e038399. doi:10.1136/ bmjopen-2020-038399

- Prepublication history and additional material are published online only. To view, please visit the journal online (http://dx.doi. org/10.1136/bmjopen-2020038399).

Received 09 March 2020 Revised 12 February 2021 Accepted 15 February 2021

Check for updates

(c) Author(s) (or their employer(s)) 2021. Re-use permitted under CC BY-NC. No commercial re-use. See rights and permissions. Published by BMJ.

For numbered affiliations see end of article.

Correspondence to

Dr Qunhua Jin;

jqhnyfy@163.com

\section{ABSTRACT}

Introduction Total knee arthroplasty (TKA) is a common and highly effective orthopaedic procedure for treating end-stage knee osteoarthritis. Tranexamic acid (TXA) has become a routine part of perioperative care in TKAs. The best practices regarding the delivery method of TXA in TKA remain controversial. Plasminogen activator inhibitor-1 (PAl-1), thrombin-antithrombin (TAT) complexes and prothrombin fragment $\mathrm{F} 1+2(\mathrm{~F} 1+2)$ have been demonstrated to be elevated in patients with venous thromboembolism (VTE). The aim of this trial was to investigate the most efficacious delivery method of TXA (comparison of intravenous and topical applications and comparison of three topical applications) and to evaluate the safety of TXA strategies by investigating the effect of TXA on the plasma D-dimer, PAl-1, TAT and F1+2 levels. Methods and design This trial is a prospective, randomised, controlled study that will evaluate the efficacy and safety of strategies of TXA. A total of 250 patients undergoing primary TKA will be randomly allocated to five groups for different TXA applications. The primary outcome is total blood loss. The secondary outcomes are blood transfusion rate, drainage volume, plasma D-dimer, PAl-1, TAT and F1+2 levels, maximum haemoglobin drop, wound complications, VTE and length of hospital stay.

Ethics and dissemination This study's protocol is in accordance with the declaration of Helsinki. The ethics committee of the General Hospital of Ningxia Medical University approved this study (approval ID: 2020-505). The results of this study will be disseminated in international peer reviewed journals.

Trial registration number ChiCTR2000030624.

\section{INTRODUCTION}

Total knee arthroplasty (TKA) is a common and highly effective orthopaedic procedure for treating end-stage knee osteoarthritis, which can reconstruct joint alignment, alleviate pain and improve joint function. It was estimated that, from 2005 to 2030, the
Strengths and limitations of this study

- This trial is the first study to investigate the most effective administration from intravenous and three topical applications

- In addition to vascular ultrasound and D-dimer, this is the first study using plasminogen activator inhibitor-1, thrombin-antithrombin and prothrombin fragment F1+2 to evaluate safety of tranexamic acid in total knee arthroplasty (TKAs).

- All TKA procedures will be performed by one surgeon, and the TKA will be performed using a single prosthetic system; therefore, the results of this study are not implant dependent, and the surgery outcomes are lowered by a single surgeon.

- The study is conducted in a high-volume tertiary A hospital, which has well-established standardised fast-track protocols for TKA, providing consistency in interventions and assessments.

- Involving five groups in this study could be a limitation.

demand for primary total knee arthroplasties is projected to grow by $673 \%$ to 3.48 million procedures. ${ }^{1}$ However, TKA involves substantial blood loss and blood transfusion requests, which can lead to transfusion-associated complications such as disease transmission and immunological reactions, increasing burden on the healthcare system. ${ }^{2-5}$

Tranexamic acid (TXA), an indirect fibrinolytic inhibitor, has become a routine part of perioperative care in primary total knee arthroplasties. ${ }^{67}$ TXA can be delivered topically, intravenously or by combined application. ${ }^{8-10}$ For the patients undergoing TKA, topical TXA can be delivered with irrigation, periarticular soft tissue injection or injection 
through drainage. ${ }^{11-13}$ The best practices regarding timing, route or dosage of TXA in TKA remain controversial, ${ }^{14} 15$ and to our knowledge, no study has investigated the comparison between intravenous administration and those three topical administrations, or between three topical administrations.

The concern of using TXA is the possibility of causing venous thromboembolism (VTE), especially for patients with a history of VTE. The most widely used method to evaluate the safety of TXA is perioperative ultrasound examination $^{1617}$ or clinical screen. Circulating markers of thrombogenesis are by-products of the activated coagulation cascade and therefore should be elevated when clot formation is occurring. The final step in the coagulation cascade is the conversion of fibrinogen into fibrin clot. Prothrombin fragment $\mathrm{F} 1+2(\mathrm{~F} 1+2)$ is produced when prothrombin is cleaved to form thrombin, which then acts on fibrinogen. Thrombin-antithrombin (TAT) complexes are formed after thrombin acts on fibrinogen to form fibrin clot and is then inactivated. ${ }^{18}$ Plasminogen activator inhibitor-1 (PAI-1) is a single-chain glycoprotein composed of 379 amino acids with a molecular weight of $47 \mathrm{kDa} .{ }^{19}$ In recent years, many investigations reported findings regarding potential associations between PAI-1 rs1799889 A/G polymorphism and VTE, ${ }^{20} 21$ a metaanalysis has supported that PAI-1 rs1799889 polymorphism may serve as one of the predisposing factors of VTE in both Caucasians and East Asians. ${ }^{22}$ Therefore, plasma D-dimer, PAI-1, TAT and F1+2 levels could be used to monitor perioperative VTE and to evaluate the safety of TXA administration.

This is the first prospective, randomised, controlled study on the efficacy and safety of various administrations of TXA on total blood loss (TBL), blood transfusion rate (BTR) and D-dimer, PAI-1, TAT and F1+2 levels of TKA.

\section{Objectives}

This study aimed to compare the efficacy of various strategies of TXA (comparison of intravenous and topical applications and comparison of three topical applications) with TBL, BTR and drainage volume.

Moreover, this work aimed to clarify the safety of TXA strategies by investigating the effect of TXA on the plasma D-dimer, PAI-1, TAT and F1+2 levels, and by comparing the wound complications, length of hospital stay (LOS), deep vein thrombosis (DVT) and pulmonary embolism (PE).

\section{METHODS AND ANALYSIS \\ Trial design}

The study is a prospective, randomised, controlled study with a 6-month follow-up involving 250 patients undergoing TKA. The study compares TBL, BTR and drainage volume between different administrations of TXA (comparison of intravenous and topical applications and comparison of three topical applications) to determine the most effective strategy of TXA. The study also investigates the safety of TXA strategies in terms of the effect of TXA on the plasma D-dimer, PAI-1, TAT and F1+2 levels, wound complications, LOS, DVT and PE. Randomisation will be performed with a 1:1:1:1:1 allocation into five groups: placebo group, intravenous group, topical irrigation (TI) group, periarticular tissue injection (PI) group and and drainage injection (DI) group.

\section{Study setting}

The study is performed in the General Hospital of Ningxia Medical University, a tertiary A hospital. All TKA procedures will be performed by one surgeon (QJ) who has previously performed a minimum of 1000 TKA procedures. A training manual will be produced to educate and train the study team, including anaesthesiologists, nurses and clinical research coordinators before starting the trial.

This trial is registered online (chictr.org.cn). The study protocol construction follows the SPIRIT $2013^{23}$ and the Consolidated Standards of Reporting Trials (CONSORT) statements. ${ }^{24}$ A CONSORT flow diagram of the trial is summarised in figure 1 .

\section{Sample size calculation}

A previous study based on an analysis of a national database with 7133 primary TKA procedures has indicated that the TBLs of intravenous TXA application, topical TXA application and control group were $830 \pm 410 \mathrm{~mL}$, $970 \pm 470 \mathrm{~mL}$ and $1200 \pm 640 \mathrm{~mL}$, respectively. ${ }^{2}$ Based on these data, the sample size was calculated by PASS V.15.0 (NCSS, LLC, Kaysville, Utah, USA), with one-way analysis of variance (ANOVA) F-tests. We calculated a total of 39 patients per group to provide a power of $80 \%$ to detect it at a significance level of $5 \%$. Considering factors such as loss of follow-up, we expanded the sample size by about $30 \%$; therefore, there will be a minimum of 50 patients in each group.

\section{Patient and public involvement}

No patients were involved in the study.

\section{Patient enrolment and eligibility criteria}

Patient enrolment in this study will occur during the preoperative outpatient visit; the decision to perform the TKA is made by the surgeon according to good clinical practice. A total of 250 consecutive eligible patients will be enrolled in this trial. A detailed list of inclusion and exclusion criteria is presented in box 1 .

\section{Randomisation, blinding and quality control}

A computer-based randomisation system will be used to screen and randomise the patients 1 day before operation. Randomisation allocation forms will be sealed in non-transparent envelopes by a person not involved in the study. The envelopes will be kept in a locked cabinet at the surgical unit. Single envelopes will be opened and thus patients will be randomised by the anaesthesia nurse no earlier than 2 hours prior to the surgery. Patients, anaesthesiologists and research assistants collecting data 


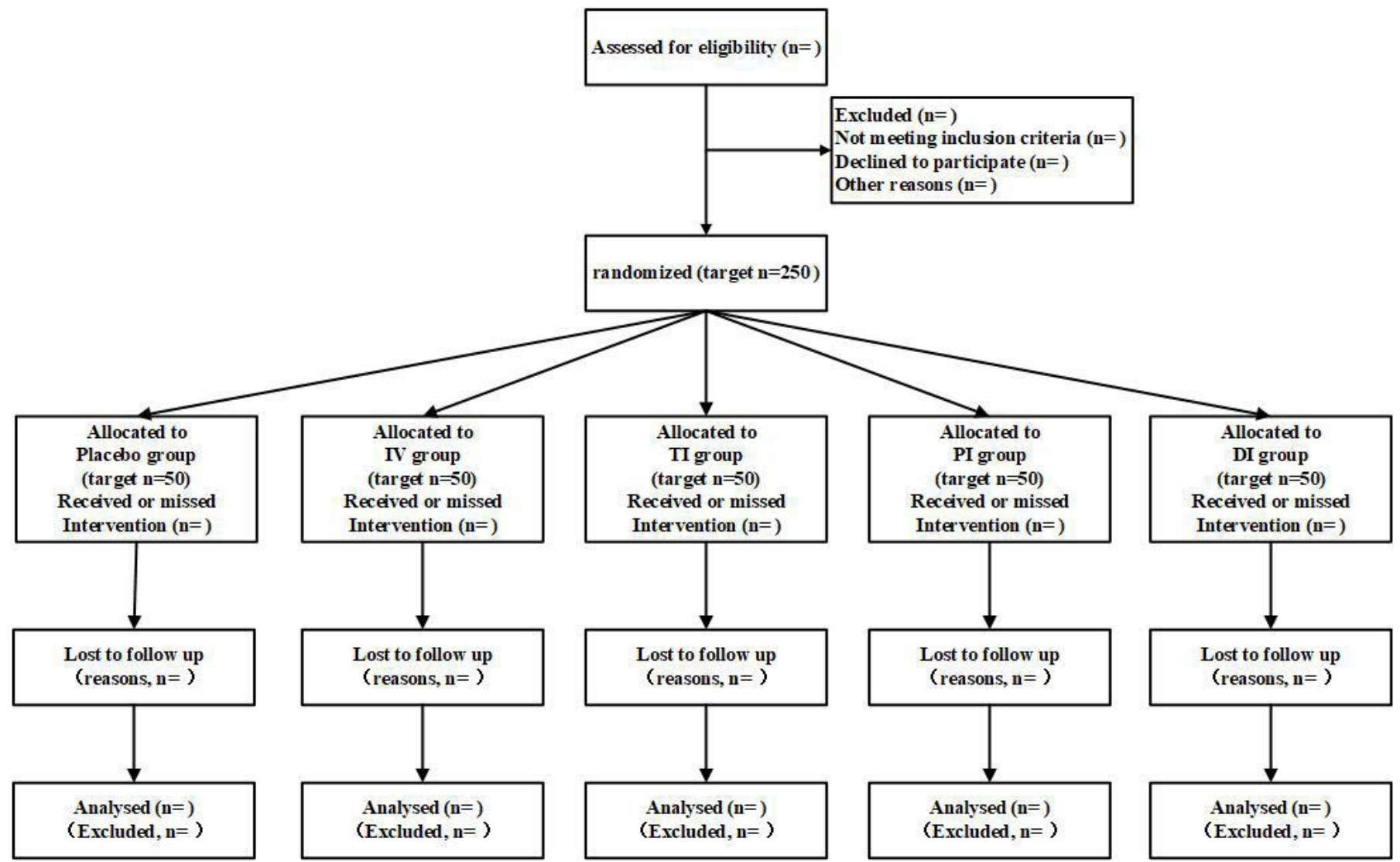

Figure 1 CONSORT flow diagram. DI, drainage injection; PI, periarticular tissue injection; TI, topical irrigation.

will be blinded to group allocation. The implementation of the study, data completeness and accuracy will be supervised by the Office of Scientific Research at General Hospital of Ningxia Medical University.

\section{Intervention}

All TKA procedures will be performed by one surgeon $(\mathrm{QJ})$, under general anaesthesia, with tourniquets. A standard midline skin incision and medial parapatellar arthrotomy approach will be used. Standard surgical

\section{Box 1 Inclusion and exclusion criteria}

\section{Inclusion criteria}

1. Undergoing primary total knee arthroplasty of both genders.

2. $>18$ and $<100$ years at the time of inclusion.

\section{Exclusion criteria}

1. Allergy to tranexamic acid.

2. Preoperative hepatic or renal dysfunction.

3. Serious cardiac or respiratory disease, including coronary artery stent placement or bypass.

4. Congenital or acquired coagulopathy, as evidenced by an international normalised ratio of $>1.4$ or a partial thromboplastin time of $>1.4$ times normal.

5. A preoperative platelet count of $<150000 / \mathrm{mm}^{3}$.

6. History of a prothrombotic condition.

7. Pregnancy or breast feeding.

8. Diagnosis of inflammatory arthritis.

9. A preoperative haemoglobin level of $<100 \mathrm{~g} / \mathrm{L}$. techniques for intraoperative haemostasis will be performed. All output will be measured and recorded in millilitres. All drains will be clamped for 2 hours and removed 24 hours after placement.

For patients in the placebo group, $100 \mathrm{~mL}$ normal saline (NS) will be applied intravenously $10 \mathrm{~min}$ prior to skin incision, and $100 \mathrm{~mL}$ NS will be applied intravenously at 3 and 6 hours postoperatively.

For patients in the intravenous group, $1 \mathrm{~g}$ of TXA in $100 \mathrm{~mL}$ NS will be applied intravenously $10 \mathrm{~min}$ prior to skin incision, and $1 \mathrm{~g}$ of TXA in $100 \mathrm{~mL}$ NS will be applied intravenously at 3 and 6 hours postoperatively.

For patients in the TI group, after cementation of the implant, $3 \mathrm{~g}$ of TXA diluted in $75 \mathrm{~mL}$ NS solution will be irrigated topically to the open joint surfaces 5 min prior to tourniquet release. The surgeon subsequently suctioned away excess study solution without touching the surrounding tissue surfaces.

For patients in the PI group, $3 \mathrm{~g}$ of TXA diluted in $75 \mathrm{~mL}$ NS solution will be injected to periarticular tissue, including the medial and lateral capsules, the quadriceps muscle tendon and the infrapatellar fat pad, prior to capsule closure.

For patients in the DI group, after wound closure, $3 \mathrm{~g}$ of TXA diluted in $75 \mathrm{~mL}$ NS will be injected into joint cavity through drainage.

As placebo, the patients in the TI, PI and DI groups will also receive $100 \mathrm{~mL}$ NS intravenously $10 \mathrm{~min}$ prior to skin incision, 3 and 6 hours postoperatively. 
VTE prophylaxis will be standardised according to the institution with low-molecular-weight heparin. All patients will also be managed with a comprehensive, multidisciplinary approach to postoperative care, including mechanical prophylaxis prior to discharge, early mobilisation with physical therapy, medical optimisation and regional anaesthesia, if appropriate. All patients will undergo ultrasound examination of the lower extremity blood vessels before discharge. Patients will be considered for blood transfusion if the haemoglobin level is less than $80 \mathrm{~g} / \mathrm{L}$ with symptoms (defined as syncope, lightheadedness, shortness of breath, fatigue and palpitations).

\section{ASSESSMENTS}

\section{Sample collection}

Peripheral blood samples will be collected at several time points: 1-3 days prior to operation, second hour, first and third days after the operation, respectively. All samples will be placed on ice and centrifuged at $3500 \mathrm{rpm}$ for $5 \mathrm{~min}$. The plasma will be frozen and stored at $-80{ }^{\circ} \mathrm{C}$. Preoperative and postoperative samples will be drawn to assess haemoglobin, haematocrit, D-dimer, PAI-1, TAT and F1+2 levels.

\section{Preoperative phase}

Medical data and history, including height, weight, allergies, tobacco use, alcohol use, ASA presurgical physical status class, historical illnesses and surgeries, together with preoperative standard laboratory tests, will be collected from every patient. Imaging examination and surgical planning will be performed for every patient.

\section{Postoperative phase}

A total drainage volume of 24 hours will be recorded for each patient. The blood volume and TBL will be calculated using the formula reported by Nadler $e t a l^{25}$ A maximum haemoglobin drop will be calculated and recorded for each patient. The patients requiring blood transfusion in each group will be recorded. The complications, such as wound complications and VTE, will also be collected. Patients will be discharged within 3-5 days, postoperatively, if no complication occurs. The LOS will also be collected. Patients will be invited to the outpatient clinic 2 weeks and 1, 3 and 6 months after the operation to assess and record the complications and mortality. The vascular ultrasonography will also be performed at the follow-up time points.

Outcome measures are presented in table 1.

\section{Study schedule}

This study began on 1 May 2020. It is estimated that this study will be completed by 30 September 2021 .

\section{Statistical analysis}

The statistical analysis will be performed by using SPSS V.19.0 software. The baseline continuous variables will be summarised using mean $\pm \mathrm{SD}$ or median (IQR) for continuous variables as appropriate. Categorical variables will

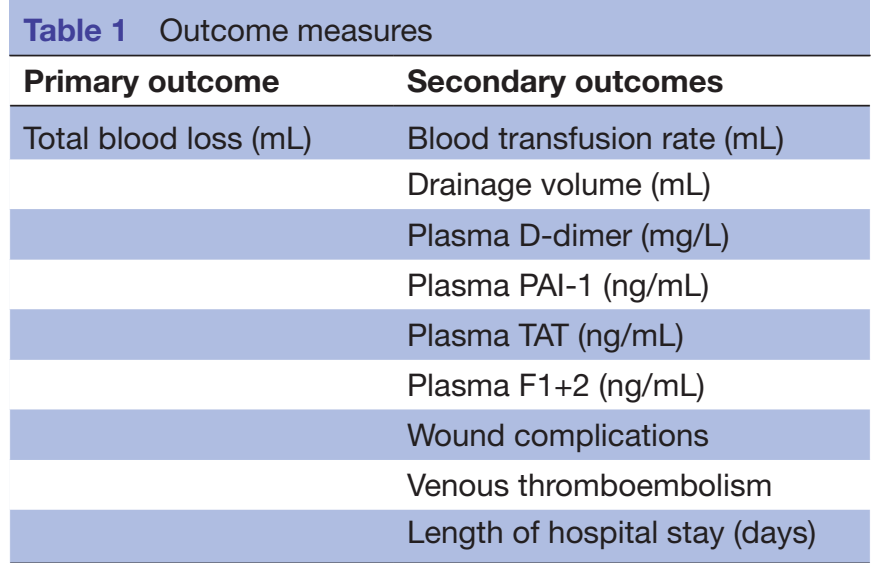

$\mathrm{F} 1+2$, prothrombin fragment $\mathrm{F} 1+2$; $\mathrm{PAl}-1$, plasminogen activator inhibitor-1; TAT, thrombin-antithrombin complexes.

be summarised using frequency (percentage). Normality of data will be tested by the Kolmogorov-Smirnov test. One-way ANOVA will be used to compare the TBL, drainage volume, plasma D-dimer, PAI-1, TAT and F1+2 levels, maximum haemoglobin drop and LOS between groups. If there is a significant difference, the comparison between groups will be performed by Scheffe post hoc analysis test. Repeated-measures ANOVA will be used to compare plasma PAI-1, TAT, F1+2 levels according to time points. $\chi^{2}$ test will be used to compare the BTR, VTE and wound complications between groups.

\section{DISCUSSION}

TKA is an effective treatment option to correct deformity, ameliorate pain and improve quality of life for advanced osteoarthritis of the knee. Since the concept of Enhanced recovery after surgery (ERAS), or fast-track surgery, was introduced in 1997 by Dr Henrik Kehlet, ${ }^{26}$ the ERAS has been widely accepted in TKA. Blood management is an inseparable part of all big orthopaedic surgeries, including TKA procedure.

TXA, an antifibrinolytic agent, has received increasing attention; it is shown to have effectively reduced blood loss following TKA. While previous studies have compared TXA administrations between intravenous usage and one or two of three topical usages, ${ }^{27-32}$ and there is no consensus or best practices regarding route of TXA in knee arthroplasty. Therefore, we designed this study to compare the efficacy of different strategies of TXA (comparison of intravenous and topical applications and comparison of three topical applications).

In this study, we conduct plasma D-dimer, PAI-1, TAT and F1+2 levels to evaluate the safety of TXA. Because the sensitivity and specificity of those three markers remain unclear in TKA, we will perform ultrasound examination of the lower extremity blood vessels for each patient to confirm the diagnosis of DVT.

In summary, this study was designed to investigate the most effective administration from intravenous and three 
topical applications, and to evaluate the safety of TXA with D-dimer, PAI-1, TAT and F1+2.

\section{Monitoring}

An orthopaedic surgeon, an anaesthesiologist, a statistician, a physician and a member from the Office of Scientific Research who are not related to this trial will be involved for the entire duration of the trial to review and ensure protocol compliance, and to make the decision to stop the trial if there are abnormal safety issues or serious complications.

\section{Ethics and dissemination}

This study protocol is in accordance with the most recent version of the World Medical Association Declaration of Helsinki. The ethics committee of General Hospital of Ningxia Medical University approved this study (approval ID: 2020-505).

\section{Consent}

Details of the study will be explained thoroughly to the potential participants by the study investigator at the preoperative interview. The informed consent form must be signed and provided by all eligible individuals before enrolment. The participants can withdraw from the study without any reason at any time. The privacy of all participants will be strictly protected. Any confidential information, such as name, identification number and hospital administration number will not be exported, and data anonymity will be executed during the process of data analysis and management.

\section{Author affiliations}

${ }^{1}$ Department of orthopedics, General Hospital of Ningxia Medical University, Yinchuan, Ningxia, China

${ }^{2}$ Clinical college, Ningxia Medical University, Yinchuan, Ningxia, China

${ }^{3}$ School of Public Health, Ningxia Medical University, Yinchuan, Ningxia, China

Contributors YY, ZW and QJ are responsible for the original study planning. YY, XZ and QJ applied for this study's funding and for the postoperative evaluations of 3 and 12 months. $\mathrm{KY}, \mathrm{JH}$ and $\mathrm{YJ}$ trained the hospital personnel. $\mathrm{KY}$, JH and YJ were responsible for patient recruitment; DD, HY and QJ were responsible for the 24hour evaluation of the patients. YY and ZW were responsible for controlling patient data deposits and checked the data input. YY, ZW and XZ were experts in outcome prediction models, intensive care and severe complications, and orthopaedic research. All authors participated in the analysis and reporting of the results. FW, as a professional statistician, was consulted for statistical analyses. YY and QJ conceived the study. All authors contributed to refining the study protocol and approving the final manuscript.

Funding This trial is supported by a grant from the Ningxia University Scientific Research Project (project no. NGY2020050) and the Ningxia key R \& D program key project: Basic Research and Clinical Application of Geriatric Orthopedic Diseases (project no. 2018BEG02005).

Competing interests None declared.

Patient and public involvement Patients and/or the public were not involved in the design, conduct, reporting or dissemination plans of this research.

Patient consent for publication Not required.

Provenance and peer review Not commissioned; externally peer reviewed.

Open access This is an open access article distributed in accordance with the Creative Commons Attribution Non Commercial (CC BY-NC 4.0) license, which permits others to distribute, remix, adapt, build upon this work non-commercially, and license their derivative works on different terms, provided the original work is properly cited, appropriate credit is given, any changes made indicated, and the use is non-commercial. See: http://creativecommons.org/licenses/by-nc/4.0/.

ORCID iD

Qunhua Jin http://orcid.org/0000-0003-1901-7905

\section{REFERENCES}

1 Kurtz S, Ong K, Lau E, et al. Projections of primary and revision hip and knee arthroplasty in the United States from 2005 to 2030. J Bone Joint Surg Am 2007;89:780-5.

2 Xie J, Hu Q, Huang Z, et al. Comparison of three routes of administration of tranexamic acid in primary unilateral total knee arthroplasty: analysis of a national database. Thromb Res 2019:173:96-101.

3 Hart A, Khalil JA, Carli A, et al. Blood transfusion in primary total hip and knee arthroplasty. incidence, risk factors, and thirty-day complication rates. J Bone Joint Surg Am 2014;96:1945-51.

4 Harvey AR, Basavaraju SV, Chung K-W, et al. Transfusion-related adverse reactions reported to the National healthcare safety network Hemovigilance module, United States, 2010 to 2012. Transfusion 2015;55:709-18.

5 Kozanek M, Menendez ME, Ring D. Association of perioperative blood transfusion and adverse events after operative treatment of proximal humerus fractures. Injury 2015;46:270-4.

6 Fillingham YA, Ramkumar DB, Jevsevar DS, et al. The efficacy of tranexamic acid in total knee arthroplasty: a network meta-analysis. J Arthroplasty 2018;33:3090-8.

7 Wong J, Abrishami A, El Beheiry $\mathrm{H}$, et al. Topical application of tranexamic acid reduces postoperative blood loss in total knee arthroplasty: a randomized, controlled trial. J Bone Joint Surg Am 2010;92:2503-13.

8 Yue C, Kang P, Yang P, et al. Topical application of tranexamic acid in primary total hip arthroplasty: a randomized double-blind controlled trial. J Arthroplasty 2014;29:2452-6.

9 Wang C, Kang P, Ma J, et al. Single-Dose tranexamic acid for reducing bleeding and transfusions in total hip arthroplasty: a double-blind, randomized controlled trial of different doses. Thromb Res 2016;141:119-23.

10 Sun Q, Li J, Chen J, et al. Comparison of intravenous, topical or combined routes of tranexamic acid administration in patients undergoing total knee and hip arthroplasty: a meta-analysis of randomised controlled trials. BMJ Open 2019;9:e024350.

11 Prakash J, Seon J-K, Park YJ, et al. A randomized control trial to evaluate the effectiveness of intravenous, intra-articular and topical wash regimes of tranexamic acid in primary total knee arthroplasty. $J$ Orthop Surg 2017;25:230949901769352.

12 Jules-Elysee KM, Tseng A, Sculco TP, et al. Comparison of topical and intravenous tranexamic acid for total knee replacement. $J$ Bone Joint Surg 2019;101:2120-8.

13 Hirose $\mathrm{H}$, Ogawa $\mathrm{H}$, Matsumoto $\mathrm{K}$, et al. Periarticular injection of tranexamic acid promotes early recovery of the range of knee motion after total knee arthroplasty. J Orthop Surg 2019;27:230949901986469.

14 Georgiadis AG, Muh SJ, Silverton CD, et al. Response to Letter to the Editor on "A Prospective Double-Blind Placebo Controlled Trial of Topical Tranexamic Acid in Total Knee Arthroplasty". J Arthroplasty 2020;35:1161-2.

15 Fillingham YA, Ramkumar DB, Jevsevar DS, et al. Tranexamic acid in total joint arthroplasty: the endorsed clinical practice guides of the American association of hip and knee surgeons, American Society of regional anesthesia and pain medicine, American Academy of orthopaedic surgeons, hip Society, and knee Society. Reg Anesth Pain Med 2019;44:7-11.

16 Tzatzairis TK, Drosos Gl, Kotsios SE, et al. Intravenous vs topical tranexamic acid in total knee arthroplasty without tourniquet application: a randomized controlled study. J Arthroplasty 2016;31:2465-70.

17 Scott AM. Total knee replacement and imaging. Radiol Technol 2015;87:65-86.

18 Su EP, Mount LE, Nocon AA, et al. Changes in markers of thrombin generation and interleukin-6 during Unicondylar knee and total knee arthroplasty. J Arthroplasty 2018;33:684-7.

19 Huber K, Christ G, Wojta J, et al. Plasminogen activator inhibitor type-1 in cardiovascular disease. status report 2001. Thromb Res 2001;103 Suppl 1:S7-19.

20 Bezgin T, Kaymaz C, Akbal Özgür, et al. Thrombophilic gene mutations in relation to different manifestations of venous 
thromboembolism: a single tertiary center study. Clin App/ Thromb Hemost 2018;24:100-6.

21 D'Amico M, Pasta F, Pasta L. Thrombophilic genetic factors PAl-1 4G-4G and MTHFR 677TT as risk factors of alcohol, cryptogenic liver cirrhosis and portal vein thrombosis, in a Caucasian population. Gene 2015;568:85-8.

22 Geng B, Li S, Zhou J, et al. Correlation between PAl-1 rs1799889 polymorphism and venous thromboembolism: A meta-analysis of 48 case-control studies. Phlebology 2020;35:268355519897552:472-9.

23 Chan A-W, Tetzlaff JM, Altman DG, et al. Spirit 2013: new guidance for content of clinical trial protocols. The Lancet 2013;381:91-2.

24 Schulz KF, Altman DG, Moher D, et al. Consort 2010 statement: updated guidelines for reporting parallel group randomised trials. Int J Surg 2011;9:672-7.

25 Nadler SB, Hidalgo JH, Bloch T. Prediction of blood volume in normal human adults. Surgery 1962;51:224-32.

26 Kehlet $\mathrm{H}$. Multimodal approach to control postoperative pathophysiology and rehabilitation. $\mathrm{Br} J$ Anaesth 1997;78:606-17.

27 Abdel MP, Chalmers BP, Taunton MJ. Intravenous versus topical tranexamic acid in total knee arthroplasty: both effective in a randomized clinical trial of 640 patients. J Bone Jt Surg 2018;100:1023-9.

28 Aguilera X, Martínez-Zapata MJ, Hinarejos P, et al. Topical and intravenous tranexamic acid reduce blood loss compared to routine hemostasis in total knee arthroplasty: a multicenter, randomized, controlled trial. Arch Orthop Trauma Surg 2015;135:1017-25.

29 George J, Eachempati KK, Subramanyam KN, et al. The comparative efficacy and safety of topical and intravenous tranexamic acid for reducing perioperative blood loss in total knee arthroplasty- a randomized controlled non-inferiority trial. Knee 2018;25:185-91.

30 Patel JN, Spanyer JM, Smith LS, et al. Comparison of intravenous versus topical tranexamic acid in total knee arthroplasty: a prospective randomized study. J Arthroplasty 2014;29:1528-31.

31 Wang D, Wang H-Y, Cao C, et al. Tranexamic acid in primary total knee arthroplasty without tourniquet: a randomized, controlled trial of oral versus intravenous versus topical administration. Sci Rep 2018;8:13579.

32 Zekcer A, Priori RD, Tieppo C, et al. Comparative study of topical $v s$. intravenous tranexamic acid regarding blood loss in total knee arthroplasty. Rev Bras Ortop 2017;52:589-95. 